\title{
Review: oral Haemophilus influenzae vaccination reduces the number and severity of recurrent bronchitis episodes
}

Foxwell AR, Cripps AW.Haemophilus influenzae oral vaccination against acute bronchitis. Cochrane Review, latest version 1 Sep 1999. In: Cochrane Library. Oxford: Update Software.

QUESTION: Does an oral, whole cell, non-typeable Haemophilus influenzae (NTHi) vaccine protect against recurrent episodes of acute bronchitis?

\section{Data sources}

Studies were identified by searching Medline (1965-98), Extramed (1994-8), ISI Current Contents (1993-8), Carl Uncover (1988-98), and the Cochrane Controlled Trials Register with the terms bronchitis, haemophilus, vaccine, bronchostat, and bucaline burna; by scanning relevant books, bibliographies of articles, and conference abstracts; and by contacting authors and experts in the field.

\section{Study selection}

Published studies were selected if they were randomised controlled trials that compared the effects of oral monobacterial NTHi vaccination with placebo in patients between 19 and 93 years of age who had recurrent exacerbations of acute bronchitis or chronic obstructive pulmonary disease; patients in vaccine and placebo groups were matched by age; and main outcomes were number of patients using antibiotics (an indication of the severity of exacerbation), bronchitis episodes, or rate of NTHi carriage in the respiratory tract.

\section{Data extraction}

2 reviewers independently extracted data on study quality and characteristics, patient characteristics, interventions, and outcomes.

\section{Main results}

6 studies (440 patients) met the selection criteria. 5 studies were done in patients with chronic bronchitis (mean age range 51 to $71 \mathrm{y}$ ), and 1 was done in patients with recurrent episodes of acute bronchitis (mean age $46 \mathrm{y}$ ). 5 studies were done in Australia, and 1 was done in New Guinea; all studies used a concealed allocation procedure; and study duration ranged from 3 to 12 months. At 6 months, fewer patients who received NTHi vaccination used antibiotics than did those who received placebo (table); no difference existed at 3 months. NTHi vaccination reduced bronchitis episodes at 3 and

\section{1}

Oral Haemophilus influenzae vaccination v placebo for recurrent bronchitis*

\begin{tabular}{llllll} 
& & \multicolumn{3}{c}{ Weighted event rates } & \\
Outcomes & Follow up & Vaccine & Placebo & RRR (95\% CI) & NNT (CI) \\
Antibiotic use & 6 months & $32 \%$ & $59 \%$ & $46 \%(13$ to 66$)$ & $4(3$ to 12$)$ \\
\hline & & & & Weighted mean decrease (CI) \\
\hline Bronchitis episodes & 3 months & & & $6.69(6.42$ to 6.96$)$ \\
\hline \multicolumn{3}{c}{6 months } & & & $4.47(4.30$ to 4.64$)$ \\
\hline
\end{tabular}

*Fixed-effects model used for all outcomes. Abbreviations defined in glossary; NNT and Cl calculated from data in article.
6 months more than did placebo (table). NTHi vaccination and placebo did not differ for rates of NTHi carriage in the respiratory tract at 3 or 6 months.

\section{Conclusion}

An oral whole cell, non-typeable Haemophilus influenzae vaccine reduces the number and severity of recurrent episodes of acute bronchitis.

\section{COMMENTARY}

Foxwell and Cripps reviewed data from 6 studies to determine whether an oral, whole cell NTHi vaccine would protect against recurrent episodes of bronchitis. The review is titled "Haemophilus influenzae oral vaccination against acute bronchitis" and appears to be misnamed. Acute bronchitis is caused by viral infection. ${ }^{1}$ The authors addressed the issue of acute exacerbations of chronic bronchitis.

A recent classification has defined 4 categories of bronchitis ${ }^{2}$ : (1) acute tracheobronchitis, which is usually of viral nature and requires no antibiotic treatment; (2) simple chronic bronchitis, which is associated with an $\mathrm{FEV}_{1}>50 \%$ of normal, increased sputum volume, and purulence (in this group, Haemophilus influenzae, Moraxella catarrhalis, and Streptococcus pneumoniae are the usual pathogens); (3) complicated chronic bronchitis, which is defined as increased sputum volume; purulence; and $\mathrm{FEV}_{1}<50 \%$, advanced age, $\geqslant 4$ exacerbations/year, or comorbid conditions (this group is associated with pathogens similar to those of category 2); and (4) chronic bronchial infection, which is defined similarly as category 3 with continuous sputum production throughout the year (possible pathogens include enterobacteriaceae and Pseudomonas aeruginosa). Without stratification for the type of bronchitis at randomisation, the results of this analysis are difficult to interpret.

Given the short term effects of this vaccine, it is probably better to focus on prevention of chronic bronchitis with antismoking campaigns aimed at teenagers and to develop a more effective strategy to enable current smokers to quit.

Thomas J Marrie, MD

University of Alberta

Edmonton, Alberta, Canada

1 Gwaltney JM Jr. Acute bronchitis. In: Mandell GL, Bennett JE, Dolin R, editors. Principles and practice of infectious diseases. 5th edition. Philadelphia: Churchill Livingstone, 2000:703-5.

2 Grossman RF. Guidelines for the treatment of acute exacerbations of chronic bronchitis. Chest 1997;112(6 suppl):310S-3S. 